\title{
Non-Neoplastic Lesions that may Mimic Epithelial Malignancies of the Endometrium: Morphological and Immunohistochemical Analysis
}

\author{
Giovanna Giordano* \\ Department of Medicine and Surgery, University of Parma, Italy
}

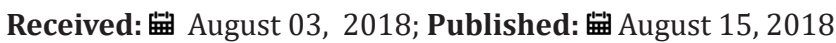

*Corresponding author: Giovanna Giordano, Department of Medicine and Surgery, Pathology Unit, University of Parma, Viale A Gramsci, 14, Parma Italy

\begin{abstract}
In this paper the author describes the morphological and immunohistochemical features of the most common benign epithelial lesions of the endometrium, which can be mistaken for neoplastic malignancies (pseudoneoplastic lesions). In addition, here it was demonstrated that both morphologic clinical data and immunohistochemical features are useful for an accurate differential pathological diagnosis from non-neoplastic lesions and epithelial malignancies.
\end{abstract}

Keywords: Endometrium; Pseudo-neoplastic lesions; Mimics of Malignancies

Abbreviations: PSN: Placental Site Nodule; PSTT: Placental Site Trophoblastic Tumor; ETT: Epithelioid Trophoblastic Tumor; CHW: Cytokeratin of High Weight; PLAP: Placental Alkaline Phosphatase; ETM: Endometrial Tubal Metaplasia; SME: Squamous Metaplasia of the Endometrium

\section{Introduction}

In this paper the author describes the morphological and immunohistochemical features of the most common benign epithelial lesions of the endometrium, which can be mistaken for epithelial malignancies (pseudo-neoplastic lesions).

\section{Materials and Methods}

The material used for this study was obtained from the archive of the Department of Medicine and Surgery, Pathology Unit, Parma University (Italy) and from a review of the literature. Relevant articles were obtained by searching the PubMed and MEDLINE databases. These databases were searched using the following key words: 'Pseudoneoplastic lesions of endometrium' and 'Mimics of malignancies of endometrium". Articles were considered if the studies included clinico-pathological and immunohistochemical features of the lesions. Papers, which were not written in English, were excluded.

\section{Main Text}

On microscopic examination, many benign alterations of the endometrium can mimic malignant epithelial lesions. Also, clinically these lesions could suggest a diagnosis of malignancy since they are characterized by abnormal uterine bleeding and can be found in an endometrial biopsy. To make a correct diagnosis, in evaluating an endometrial biopsy specimen, adequate clinical data are important, such as the age of the patient, the reason for the biopsy, the menopausal status, the use of exogenous hormones, and a history of previous pregnancy or abortion. Placental site nodules [1-3] and endometrial epithelial metaplasia [4-6] represent the most common lesions that can be misdiagnosed as malignant epithelial lesions in an endometrial biopsy. A Placental Site Nodule (PSN) is a remnant of intermediate trophoblast (extravillous trophoblast, EVT) from a previous pregnancy. Thus, it may be considered retained placental tissue in utero which does not undergo involution after pregnancy and may be detected many months or even several years after a previous pregnancy $[1-3,7]$.

The lesion represents a degenerative process of EVT that mainly involves the endometrium [3], usually, it is benign, and once removed, does not require any further treatment, and does not recur. It is an incidental finding observed in the evaluation of uterine bleeding or post-coital bleeding [1]. In the endometrium, PSN is a very small lesion, of a microscopic size, but when grossly evident, it appears as a small yellowish or tan-colored surface nodule [2-3]. Microscopically, PSN shows extensive hyalinization and cells of a different size with indistinct outlines which are organized in small groups, singly (Figure 1a). Characteristically, although there is nuclear atypia, mitotic figures are absent (Figure 1b). This lesion may mimic aggressive intermediate trophoblastic lesions, such as a placental site trophoblastic tumor (PSTT) or an epithelioid trophoblastic tumor (ETT), but also non-trophoblastic malignant neoplasms, such as squamous carcinoma. 


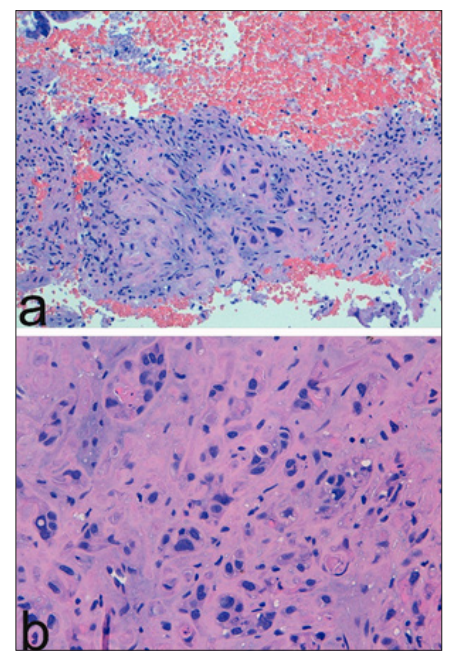

Figure 1: Placental Site Nodule PSN showing extensive hyalinization and cells of a different size with indistinct outlines which are organized in small groups, singly (a: $\mathrm{H} / \mathrm{E} \times 100)$. Note nuclear atypia and absence of mitotic figures (a: H/E x 400).

PSN must be differentiated from ETT and PSTT, because these lesions are neoplasms with a potential for local invasion and metastasis and require a different therapeutic approach. Unlike PSN, ETT is a large solid/cystic lesion that microscopically resembles a carcinoma due to the presence of cords, nests, and sheets containing hyaline material and necrotic debris and is usually clinically associated with elevated beta-HCG (Human chorionic gonadotropin). PSTT is composed of neoplastic implantation site intermediate trophoblastic cells, which are monomorphic. Like ETT, this neoplasm is a large lesion, evident macroscopically. Moreover, it can be associated with malignant behavior, and its cells invade, singly or in cords and nests, the muscle fibers of the myometrium causing tissue destruction and necrosis. Unlike PSN, PSTT lacks extensive hyalinization, and shows higher cellularity and a higher Ki-67 index [2-3].

PSN may mimic primary invasive squamous carcinoma in a biopsy specimen containing small fragments of cervical tissue [78] since the nuclear atypia and hyalinized stroma can be mistaken for keratin. Unlike squamous carcinoma, in PSN, mitoses are absent. Immunohistochemical analysis is very useful to differentiate a PSN from squamous cell carcinoma. In fact, squamous cell carcinoma shows immunoreactivity to Cytokeratin of High Weight (CHW), high index Ki 67, plus negativity to CK8 and placental alkaline phosphatase (PLAP). On the contrary, in immunohistochemical studies, a PSN reveals positivity to PLAP and CK8 (Figure 2a), negativity to CHW (Figure 2b), low Ki-67 index (Figure 2c) [8]. Endometrial epithelial metaplasia is due to hormonal or irritative stimuli. In some instances, endometrial epithelial metaplasia is related to unopposed estrogen stimuli, but in other cases, it may have a mutational origin and present glandular complexity and atypia [6]. The most common types of Endometrial epithelial metaplasia are mucinous change, papillary syncytial change, endometrial tubal metaplasia, hobnail/clear-cell/ Arias-Stella change and squamous metaplasia.

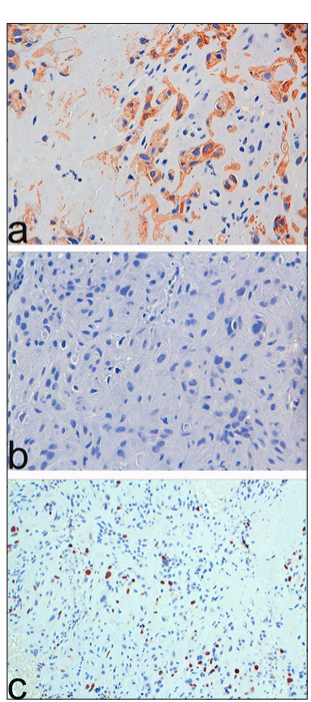

Figure 2: On immunohistochemical analysis Placental Site Nodule showing positivity to CK8 (a: $x$ 400), negativity to Cytokeratin High Weight (b: x400), low Ki-67 index (c: $x$ 200).

Mucinous metaplasia may be a focal or diffuse lesion in the endometrial mucosa. Extensive mucinous metaplasia is responsible for an uncommon condition defined as myxometra. Mucinous metaplasia may arise as a consequence of hormonal stimuli, but also as result of genetic mutation, especially in patients affected by Putz-Jeghers syndrome [6] and in this instance, it affects several organs of the female genital tract. In the complex subtype, the glandular structures may have crowded architecture, irregular glands, and a cribriform pattern. In these cases, a differential diagnosis of endometrial complex mucinous metaplasia and mucinous adenocarcinoma can be extremely difficult, if not impossible, on endometrial biopsy, since both lesions show minimal atypia and rare mitotic figures [6]. Papillary syncytial change in the endometrium, named also "eosinophilic syncytial change" can be considered as a reparative response occurring after breakdown, although its significance is yet unknown. However, it could also be a consequence of endometrial regeneration secondary to shedding, or a degenerative phenomenon due to ischemia accompanying the endometrial breakdown [6].

This lesion shows syncytial aggregates of eosinophilic cells on the endometrial surface that may extend into the glandular epithelium, forming papillary structures or micro-papillae (Figure 3a). When syncytial metaplasia is florid, this may be suggestive of a serous carcinoma or an EIC. In this circumstance, attention should be paid to the background endometrium, which, in papillary syncytial metaplasia, usually shows features of breakdown in the stroma, such as acute inflammation and fibrin thrombi (Figure $3 b)$. In addition, the epithelium of papillary syncytial change lacks nuclear atypia and high mitotic figures which are features of serous endometrial carcinoma. Endometrial tubal metaplasia (ETM) has been observed in cases associated with unopposed estrogen levels [6], and this entity can be simple or complex. The simple subtype is characterized by isolated tubal glands lined by ciliary cells. A complex subtype of ETM may be misdiagnosed as endometrial 
carcinoma, since this lesion can show cribriform glands (Figure 4) and/or papillary structures resembling endometrioid villoglandular carcinoma or serous carcinoma, respectively (Figure 5a).

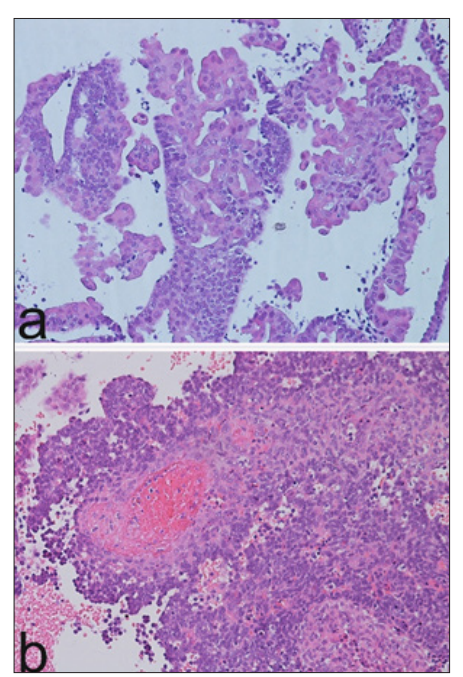

Figure 3: Endometrial syncytial metaplasia with papillary syncytial change. Note absence of nuclear atypia and mitotic figures (a: H/E $\times 200)$ and in the endometrial stroma the presence of fibrin thrombi (a: H/E x 200).

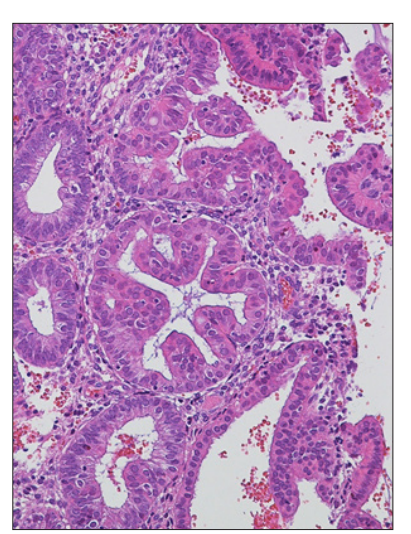

Figure 4: Complex subtype of Endometrial tubal metaplasia with cribriform glands (H/E x200).

Unlike complex ETM, serous carcinoma shows nuclear atypia, and high mitotic figures. Moreover, on immunohistochemical analysis, complex ETM is characterized by low/absent Ki-67 index, low/absent p53 expression, focal bcl-2 positivity in the intercalary and secretory cells [6] (Figure $5 \mathrm{~b}$ ) and mosaic $\mathrm{p} 16$ positivity (Figure 5c). Hobnail/clear-cell/ Arias-Stella change may be observed in the endometrium following a spontaneous abortion, in cases of extrauterine pregnancy, or in an endometrium associated with the use of a Mirena coil [9]. In abortion, Hobnail/clear-cell/ Arias-Stella change may be mixed with fragments of placental or fetal tissue and decidua. Morphologically, an Arias-Stella change is characterized by the presence of glands lined by epithelium with clear cytoplasm, nucleomegaly, exaggerated tufting and hobnail growth. In the absence of a history of pregnancy or abortion, Hobnail/clear-cell/ Arias-Stella may be mistaken for clear-cell carcinoma. However, clear-cell carcinoma can be differentiated from Hobnail/clear-cell/
Arias-Stella change because of mitotic activity, a higher degree of nuclear atypia and evident nucleoli, which are absent in a Hobnail/ clear-cell/ Arias-Stella change.

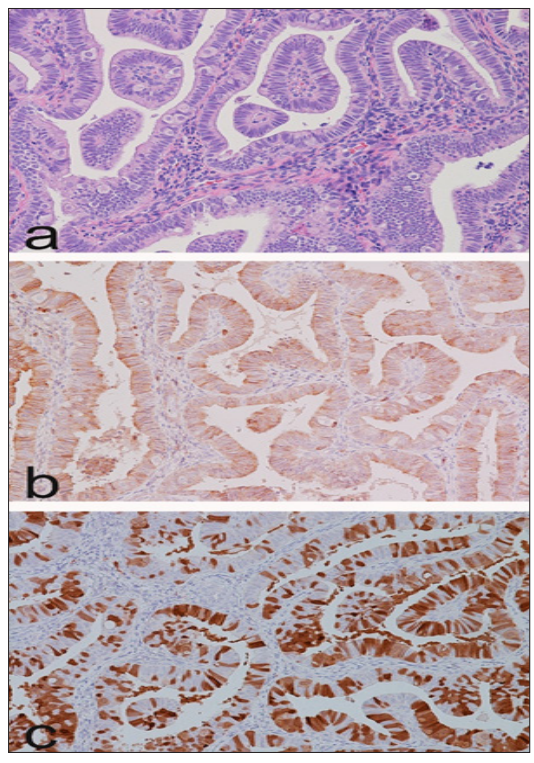

Figure 5: Complex subtype of Endometrial tubal metaplasia with papillary structures resembling endometrioid carcinoma or serous carcinoma, respectively. Note the absence of nuclear atypia and mitotic figures (a: H/E x 200). Note focal bcl-2 positivity (b. X 200) and mosaic p16 positivity (c: $x 200)$.

In addition, clear-cell carcinomashows strong and diffusenuclear positivity to Hepatocyte Nuclear Factor 1-beta (HNF-1-beta), which is absent in Hobnail/clear-cell/ Arias-Stella change [10]. Squamous metaplasia of the endometrium (SME) refers to the replacement of the endometrial surface epithelium or endometrial glands with mature squamous epithelium. SME can be due to chronic irritative stimuli, such as chronic endometritis, embolization of leiomyoma, or Vitamin A deficiency. This lesion must be differentiated from lesions that represent an extension of squamous cervical lesions to endometrial mucosa, such as verrucous carcinoma, cervical condyloma and rarer primary squamous endometrial carcinoma [11]. Also, SME may be differentiated from morular metaplasia, which is characterized by presence of morulae which are a wellcircumscribed aggregate of monomorphic spindle cells, with empty nuclei containing biotin and eosinophilic cytoplasm.

These aggregates replace part of the glandular endometrial epithelium and protrude into the glandular lumen (Figure 6a). Often, morulae are associated with complex atypical hyperplasia or may be observed in endometrial atypical polypoid adenomyoma [6], but are absent in chronic endometritis, and the embolization of leiomyoma. For accurate diagnosis it is important to bear in mind, especially in evaluating an endometrial biopsy specimen, that on immunohistochemical analysis, morular metaplasia shows immunoreactivity to markers, which are negative in both SME and squamous malignancies, such as CD 10, CDX2 and beta catenin. Characteristically, positivity to CD10 in morular metaplasia (Figure 6b) is diffuse and membranous, whereas CDX2 and beta 
catenin present nuclear positivity [6]. Because of the positivity to neuroendocrine markers, such as neuron specific enolase, synaptophysin and chromogranin, morular metaplasia should also be differentiated from neuroendocrine carcinoma [6].

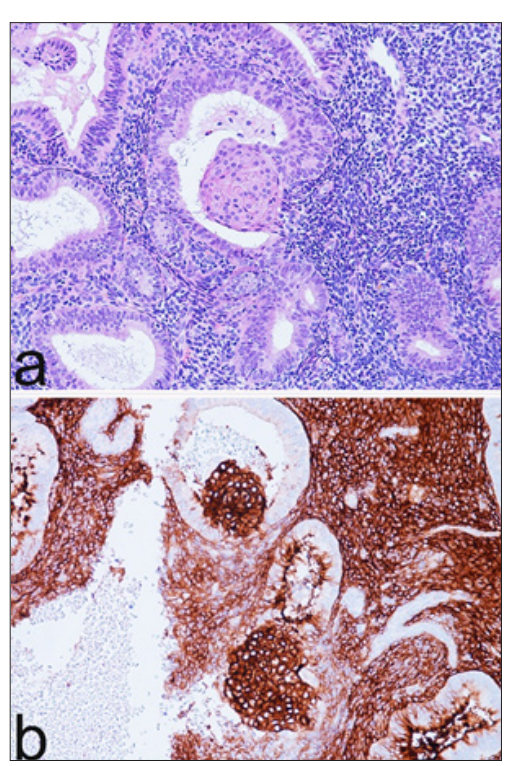

Figure 6: Morulae in morular endometrial metaplasia which characteristically present as well-circumscribed aggregate of monomorphic replacing part of the glandular endometrial epithelium, protruding into the glandular lumen (a: H/E x 200) and showing diffuse and membranous positivity to CD10 (b: x 200).

\section{Conclusion}

In conclusion, in the endometrium many benign lesions can mimic epithelial malignancies, and here it was demonstrated that both morphologic clinical data and immunohistochemical features are useful for an accurate differential pathological diagnosis.

\section{References}

1. Jacob S, Mohapatra D (2009) Placental site nodule: A tumor-like trophoblastic lesion. Indian J Pathol Microbiol 52: 240-241.

2. Benirschke K, Burton GJ, Baergen RN (2012) Trophoblastic neoplasms. In: Benirschke K, Burton GJ, Baergen RN (Eds.). Pathology of human placenta. ( $6^{\text {th }}$ Edn.). Berlin: Springer-Verlag p.723.

3. Characterization of distinctive types of intermediate trophoblast. Hum Pathol 30: 687-694.

4. McCluggage WG (2003) Metaplasias in the female genital tract. In: Lowe D, Underwood J (Eds.). Recent advances in histopathology. Vol. 20 Royal Society of Medicine Press Ltd, London, UK.

5. Hendrickson MR, Kempson RL (1980) Endometrial epithelial metaplasias: proliferations frequently misdiagnosed as adenocarcinoma. Report of 89 cases and proposed classification. Am J Surg Pathol 4: 525542.

6. Nicolae A, Preda 0, Nogales FF (2011). Endometrial metaplasias and reactive changes: a spectrum of altered differentiation. J Clin Pathol 64: 97-106.

7. Luna DV, Dulcey I, Nogales FF (2013) Coexistence of placental site nodule and cervical squamous carcinoma in a 72-year-old woman. Int J Gynecol Pathol 32: 335-337.

8. Giordano G, Manuguerra R, Varotti E, Brigati F (2016) A case of placental site nodule associated with cervical high-grade squamous intraepithelial lesion. Eur J Gynaecol Oncol 37: 259-261.

9. Hejmadi RK, Chaudhri S, Ganesan R, Rollason TP (2007) Morphologic changes in the endometrium associated with the use of the mirena coil: a retrospective study of 106 cases. Int J Surg Pathol 15: 148-154.

10. Němejcová K, Tichá I, Kleiblová P, Bártů, M, Cibula D, et al. (2016) Expression, Epigenetic and Genetic Changes of HNF1B in Endometrial Lesions. Pathol Oncol Res 22: 523-530.

11. Giordano G, D'Adda T, Merisio C, Gnetti L (2005) Primary squamous cell carcinoma of the endometrium: a case report with immunohistochemical and molecular study. Gynecol Oncol 96: 876-879.

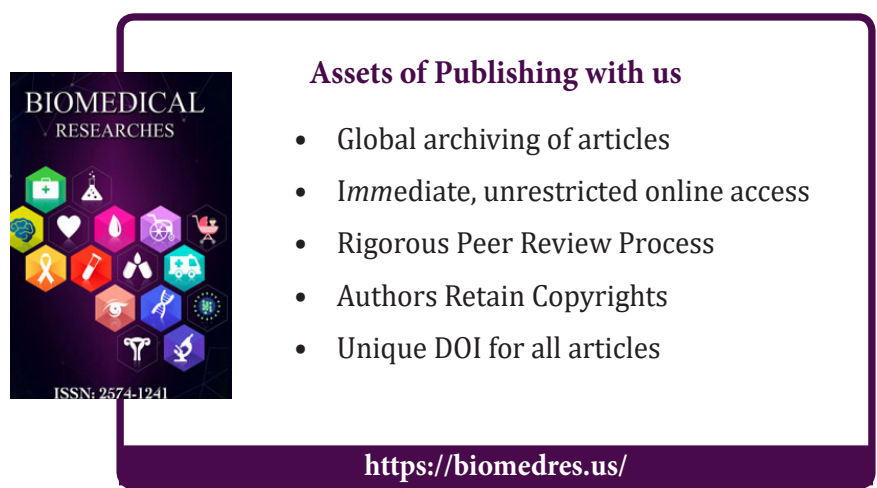

https://biomedres.us

\section{ISSN: 2574-1241}

DOI: 10.26717/BJSTR.2018.08.001588

Giovanna Giordano. Biomed J Sci \& Tech Res

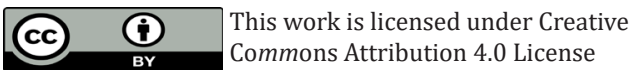

Submission Link: https://biomedres.us/submit-manuscript.php 